\title{
Epilepsy Mimicking Affective Disorder in a Patient with Amygdala Enlargement
}

\section{Case Report}

Journal of Epilepsy Research PISSN 2233-6249 / elSSN 2233-6257

Received March 29, 2019

Revised June 14, 2019

Accepted June 25, 2019

Corresponding author: Jung-Ju Lee, $\mathrm{MD}, \mathrm{PhD}$ Department of Neurology, Nowon Eulji

Medical Center, Eulji University College of

Medicine, 68 Hangeulbiseok-ro, Nowon-gu,

Seoul 01830, Korea

Tel. +82-2-970-8312

Fax. $+82-2-970-8862$

E-mail; sss331@eulji.ac.kr

\author{
Jung-Ju Lee, MD, PhD, Kyusik Kang, MD, PhD, Jong-Moo Park, MD, PhD, \\ Woong-Woo Lee, MD, PhD, Ohyun Kwon, MD, Byeong-Kun Kim, MD, PhD \\ Department of Neurology, Nowon Eulji Medical Center, Eulji University College of Medicine, Seoul, Korea
}

\begin{abstract}
Affective disorders are commonly associated with epilepsy. Affective symptoms rarely occur concomitantly with seizure occurrence, which can lead to misdiagnosis. Here, we describe a 69-year-old man who experiencedintermittent manifestations of unpleasant mood and aggressive behavior. He had temporal lobe epilepsy with amygdala enlargement. After successful treatment with an antiepileptic drug, hissymptoms resolved.Additionally, the amygdala enlargement decreased when checked at 5 years after treatment. We discuss the clinical characteristics and differential points of the case. (2019;9: 83-86)
\end{abstract}

Key words: Epilepsy, Amygdala

\section{Introduction}

Affective disorders, including depression, bipolar disorder, and anxiety disorder, are common in patients with epilepsy. Emotional symptoms associated with these disorders are typically found independent of seizure occurrence; thus, they are known asinterictal affective disorders. Patients may rarely experience symptoms of affective disorder exclusively around the time of seizure occurrence: either preceding the seizure (preictal); following the seizure (postictal); or as an expression of the seizure (ictal). ${ }^{1}$ In such situations, symptoms of affective disorders comprise the presenting symptoms of epilepsy and should be differentiated from symptoms caused by affective disorders. Previous studies have shown that temporal lobe epilepsy (TLE) with amygdala enlargement (AE) (TLE-AE) is often associated with ictal and interictal emotional disturbances. ${ }^{2-4}$ Herein, we describe a patient with TLE-AE who was initially misdiagnosed with affective disorder. The ensuing discussion addresses the clinical characteristics and differential points to distinguish emotional disturbances in TLE-AE from other psychiatric disorders.

\section{Case}

A 69-year-old man with no previous illness visited the department of psychiatry of the authors' hospital with intermittent development of an unpleasant feeling. This symptom was typically followed by ag- gressive behaviors, such as cursing and fighting with his acquaintances, which lasted for only a few minutes. He could partially recall the events after they occurred but did not understand why they had occurred. Notably, his symptoms had developed after a particularly stressful event: he had been blackmailed by an acquaintance regarding a financial issue. He was initiallydiagnosed with atypical depression and had been treated with antidepressants and antipsychotics (amisulpride $50 \mathrm{mg} /$ day and escitalopram $10 \mathrm{mg} /$ day) for 1 year; however, he did not exhibit improvement.

Subsequently, as the patient preached a sermon in church, he lost consciousness, which was preceded by the unpleasant feeling. He was then referred to the department of neurology. During meticulous history taking, his family explained that the aggressive behaviors were sometimes observed after a brief duration of behavioral arrest with a motionless stare. Electroencephalography (EEG) and magnetic resonance imaging (MRI) were performed. Epileptiform discharges were observed in the left temporal region on EEG (Fig. 1), and enlargement of the left amygdala was observed with slight hyperintensity on T2-weighted MRI (Fig. 2) without enhancement.

The symptoms completely disappeared after treatment with an antiepileptic drug (topiramate $100 \mathrm{mg} /$ day). Antidepressants and antipsychotics were gradually tapered and discontinued 6 months later. The patient has been symptom-free for 5 years. Follow-up MRI revealed decreased left amygdala volume, compared with that in previous images (Fig. 3). 


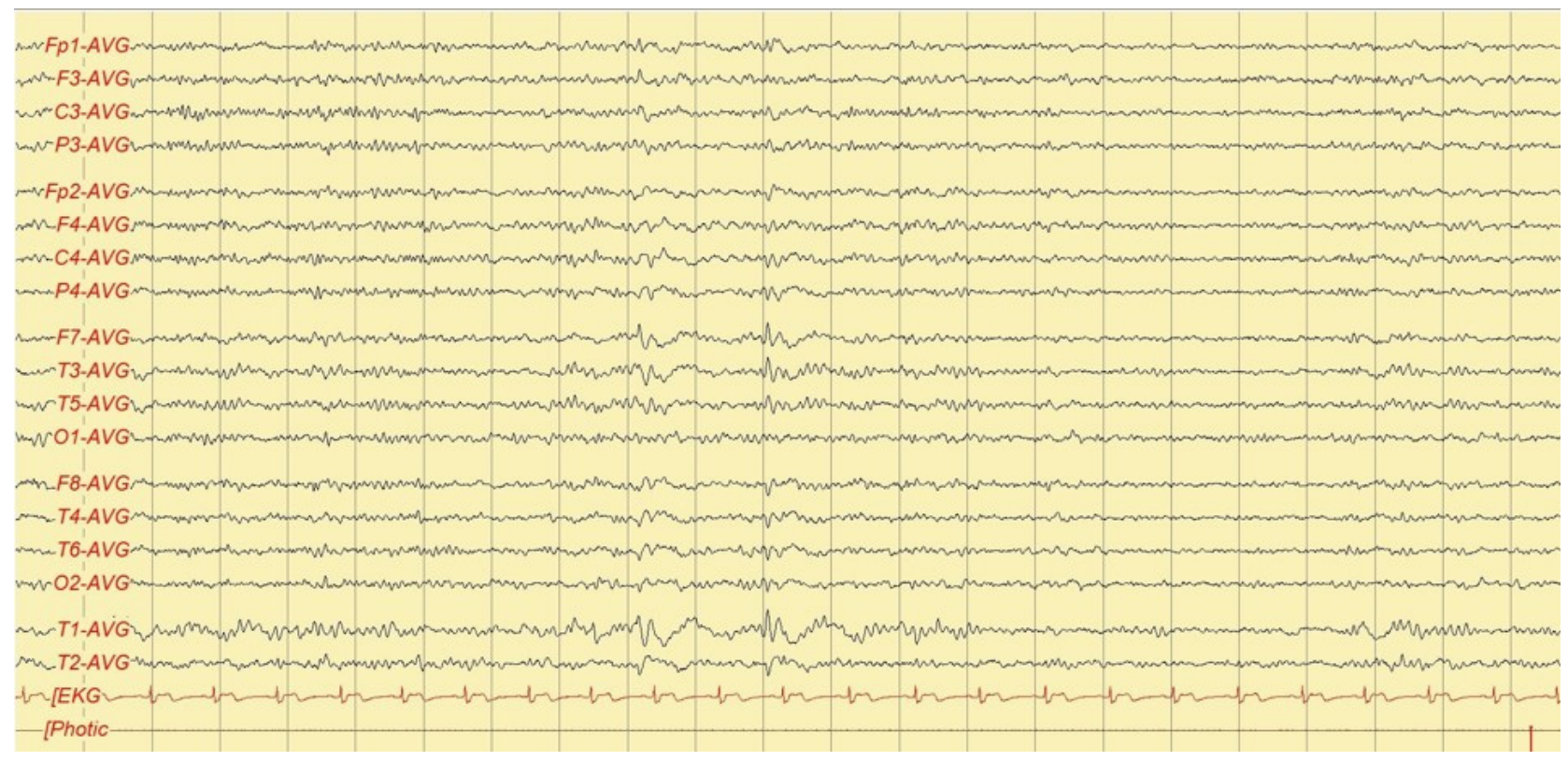

Figure 1. Electroencephalogram revealing epileptiform discharges in the left temporal leads.
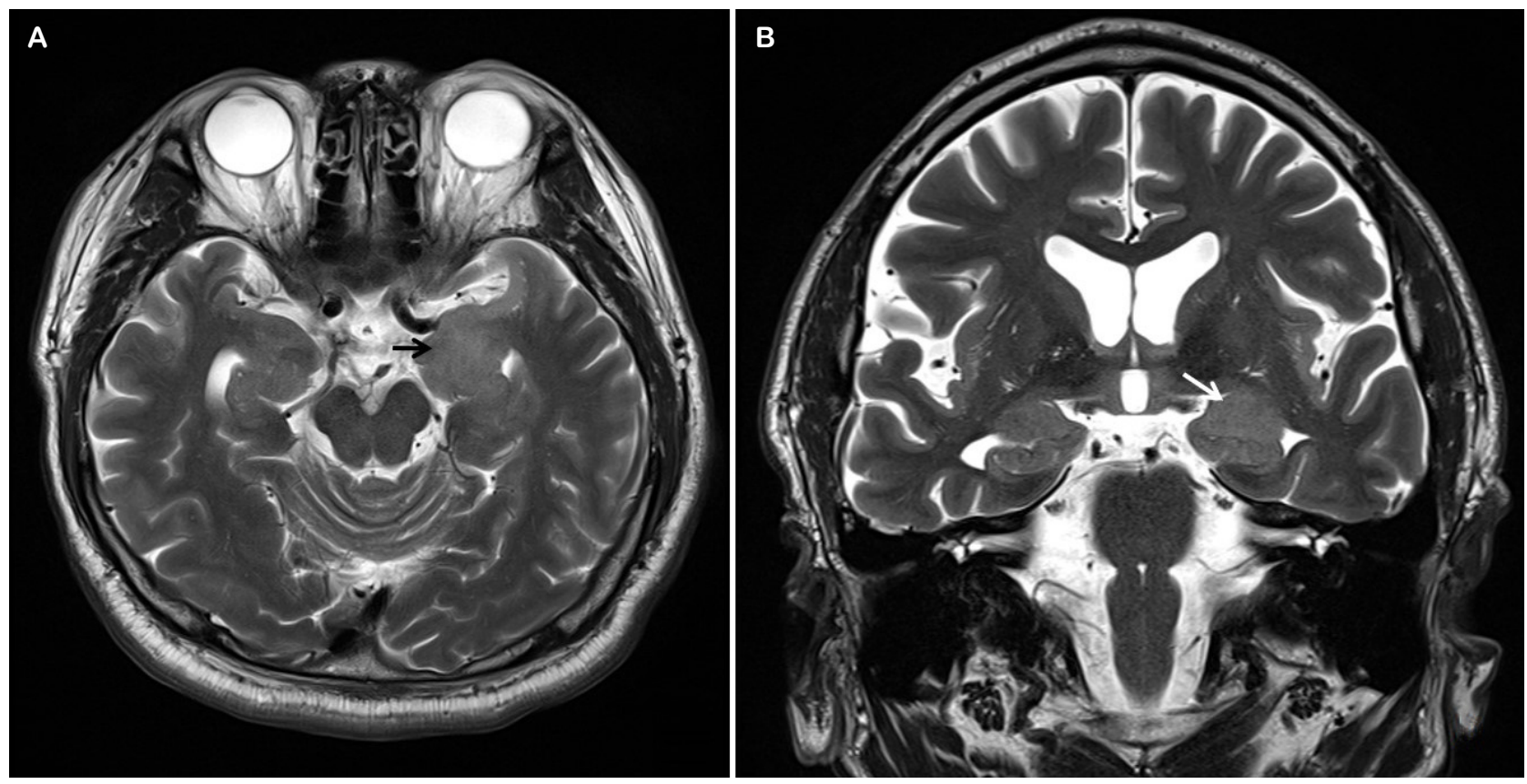

Figure 2. Axial (A) and coronal (B) T2-weighted magnetic resonance imagingrevealing increased signals in the left amygdala with enlargement (black and white arrow).

\section{Discussion}

Affective disorders, such as depression, are the most common disorders among patients visiting clinics. ${ }^{5,6}$ Rarely, affective symptoms develop as a consequence of organic brain disorders. In the patient described, emotional seizures occurred after a stressful event, which led to a misdiagnosis of a psychiatric disorder. In patients with TLE, partial preservation of consciousness during seizure can occur. ${ }^{7,8}$ Meticulous history-taking and adequate diagnostic work-up are both necessary to ensure correct diagnosis. 


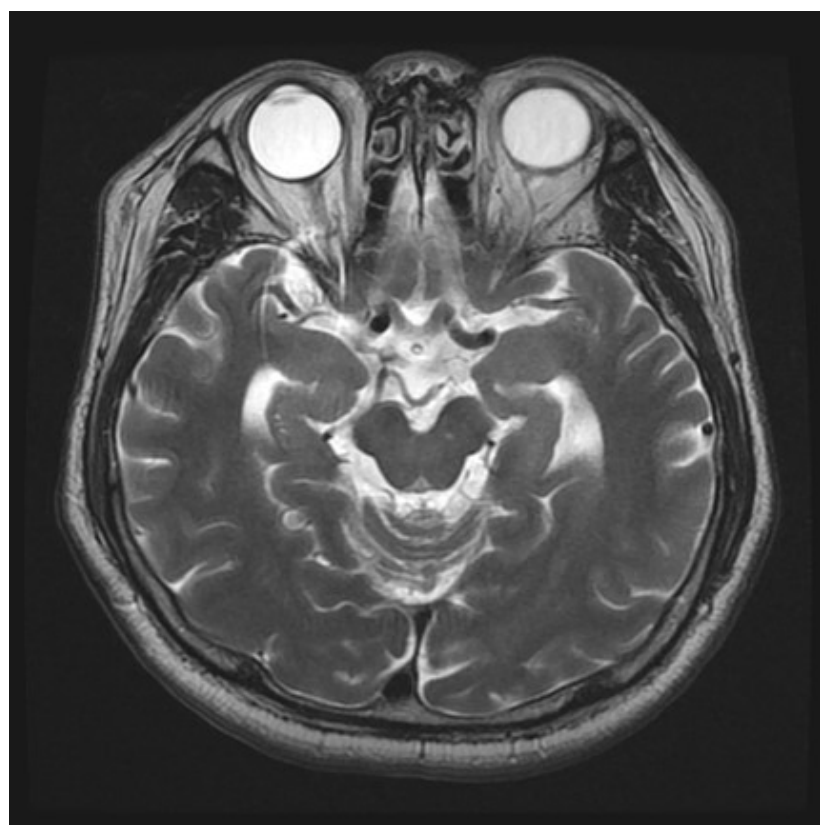

Figure 3. Follow-up magnetic resonance imagingdemonstratingthat the size of the amygdala has decreased compared with that depicted in previous images.

Epilepsy often can be misdiagnosed as a mood disorder in patients with emotional seizures without convulsive seizures. Emotional symptoms may occur as preictal, ictal, or postictal. ${ }^{1}$ In the patient described in the present case, his unpleasant mood may have been a preictal or ictal symptom, while his aggressive behavior may have been a postictal symptom. Video-EEG monitoring during seizure events, however, would be necessary to confirm our hypothesis.

The amygdala is often the focus for the initiation of seizures, or the area responsible for their propagation. ${ }^{2-4}$ Activation of the amygdala during seizures can generate various emotional symptoms. Moreover, the amygdala plays a role in interictal mood disorders. ${ }^{3} \mathrm{~A}$ previous study reported that some patients with TLE-AE treated with antiepileptic drugs experienced recovery of emotional responses, as well as control of seizures. ${ }^{2}$ In that study, TLE-AE was often associated with autoantibody-positive limbic encephalitis, as well as with focal cortical dysplasia or tumors of the amygdala. Although autoimmune pathology could be a possible etiology in our case, our patient did not exhibit any signs of autoimmune limbic encephalitis, such as hyponatremia, recurrent facio-brachial dystonic seizures, or autonomic dysfunction. Moreover, AE evanesces with anti-epileptic drug treatment rather than immunosuppressive therapy. Although the possibility of a tumor in the amygdala cannot be excluded, it is very low. However, it should be noted that some types of highly epi- leptogenic brain tumors occur frequently in the mesial temporal lobes, including the amygdala, and tend to lack radiological features of tumors that investigators attempt to screen for; as such, it cannot completely rule out the possibility of several neoplastic causes of AE.

The cause of AE is a matter of debate. Some reports have suggested that TLE-AE is a distinctive nosological and less homogeneous syndrome, and that it may be a subtype of TLE without hippocampal sclerosis. Focal cortical dysplasia and low-grade tumors or chronic inflammatory processes, including autoimmune mechanisms, can lead to $A E{ }^{2,9,10}$ Alternatively, AE may be caused by a seizure-related changes, such that chronic epileptic activity could lead to the development of AE. ${ }^{11} \mathrm{AE}$ can be reversed simply by suppressing seizures. Quantitative analysis, such as amygdala hippocampal volumetry, would have provided more objective numerical data. We did not perform quantitative analysis, which can be considered a limitation of the present case study.

In conclusion, emotional symptoms mimicking affective disorders can be a manifestation of seizure in patients with TLE-AE. AE is caused by diverse pathological disease entities and may be reversible when seizure control is achieved. Meticulous historytaking and diagnostic work-up arecritical to reach the correct diagnosis.

\section{References}

1. Mula M, Monaco F. Ictal and peri-ictal psychopathology. Behav Neurol 2011;24:21-5.

2. Holtmann O, Schlossmacher I, Moenig C, et al. Amygdala enlargement and emotional responses in (autoimmune) temporal lobe epilepsy. Sci Rep 2018:8:9561.

3. Yilmazer-Hanke D, O'Loughlin E, McDermott K. Contribution of amygdala pathology to comorbid emotional disturbances in temporal lobe epilepsy. J Neurosci Res 2016;94:486-503.

4. Van Paesschen W, King MD, Duncan JS, Connelly A. The amygdala and temporal lobe simple partial seizures: a prospective and quantitative MRI study. Epilepsia 2001;42:857-62.

5. McQuaid JR, Stein MB, Laffaye C, McCahill ME. Depression in a primary care clinic: the prevalence and impact of an unrecognized disorder. J Affect Disord 1999;55:1-10.

6. Kessler RC, McGonagle KA, Zhao S, et al. Lifetime and 12-month prevalence of DSM-III-R psychiatric disorders in the United States. Results from the National Comorbidity Survey. Arch Gen Psychiatry 1994;51:8-19.

7. Ebner A, Dinner DS, Noachtar S, Lüders H. Automatisms with preserved responsiveness: a lateralizing sign in psychomotor seizures. Neurology 1995;45:61-4.

8. Park SA, Heo K, Koh R, Chang JW, Lee BI. Ictal automatisms with preserved responsiveness in a patient with left mesial temporal lobe 
86 Journal of Epilepsy Research Vol. 9, No. 1, 2019

epilepsy. Epilepsia 2001;42:1078-81.

9. Mitsueda-Ono T, Ikeda A, Inouchi $M$, et al. Amygdalar enlargement in patients with temporal lobe epilepsy. I Neurol Neurosurg Psychiatry 2011;82:652-7.

10. Lv RJ, Sun ZR, Cui T, Guan HZ, Ren HT, Shao XQ. Temporal lobe epilepsy with amygdala enlargement: a subtype of temporal lobe epilepsy. BMC Neurol 2014;14:194.

11. Matsudaira T, Omote $Y$, Terada $T$, et al. Reversible amygdala enlargement: a longitudinal observation of a patient with elderly onset temporal lobe epilepsy. J Neurol 2017;264:2487-90. 